\title{
The importance of open-boundary estimation for an Indian Ocean GCM-data synthesis
}

\author{
by Kate Q. Zhang ${ }^{1}$ and Jochem Marotzke ${ }^{2}$
}

\begin{abstract}
The Indian Ocean general circulation is estimated by fitting the MIT Ocean General Circulation Model to the annual mean climatological hydrography and surface forcing, using the model and its computer-generated adjoint. Open boundary conditions are implemented to the west of the Indonesian Archipelago and near 30S. The approach simultaneously optimizes the initial conditions of the hydrographic fields, surface fluxes, and the open boundary conditions (temperature, salinity, and horizontal velocities).

Compared to previous results obtained in a closed domain, the estimated velocity field shows a marked improvement near the southern boundary, with a reasonably strong Agulhas Current leaving the model domain. The Indonesian throughflow (ITF) is estimated as $2.7 \mathrm{~Sv}$ ( $1 \mathrm{~Sv}$ is $10^{6} \mathrm{~m}^{3} / \mathrm{s}$ ) westward, which is on the low end of the range of previous estimates. The model is able to sharpen fronts in surface salinity, compared to climatology, and suggests that the low surface salinity values in the eastern equatorial region arise from advection out of the Bay of Bengal rather than from the ITF.

Consistent with the closed-domain results, the meridional overturning is dominated by a shallow (above $500 \mathrm{~m}$ ), wind-driven cell of $16 \mathrm{~Sv}$ maximum, which carries the bulk of the southward heat transport. We have defined generalizations of meridional heat and freshwater transports appropriate in the presence of a throughflow. The estimated meridional heat transport has a maximum of $0.8 \mathrm{PW}$ at $12 \mathrm{~S}$, maximum freshwater transport is $0.29 \mathrm{~Sv}$ southward at $9 \mathrm{~S}$. The meridional transport divergences are well balanced by the surface heat and freshwater fluxes, indicating near-steady state and small influence of the ITF.
\end{abstract}

\section{Introduction}

The formulation of open boundary conditions is crucial for every regional (that is, less than global) model that is run over sufficiently long time to allow the boundary conditions to influence the interior appreciably. The majority of general circulation models (GCMs) ${ }^{3}$ close the boundaries and employ Newtonian damping to mimic water mass conversion outside the model domain and to absorb reflections off the wall, hence forming a "sponge layer" next to the boundary (see, for example, Klinck, 1995 and Döscher et al., 1994 for

1. Division of Geological and Planetary Sciences, California Institute of Technology, Pasadena, California, 91125, U.S.A.

2. Center for Global Change Science, Department of Earth, Atmospheric and Planetary Sciences, Massachusetts Institute of Technology, Cambridge, Massachusetts, 02139, U.S.A.email: jochem@sound.mit.edu

3. Note that GCM is also used to denote "global circulation model" in meteorology. 
discussions of the sponge layers in the North Atlantic Community Modeling Effort, CME). The alternative is open boundary conditions, in which mass and properties are allowed to enter and leave the model domain, and waves are allowed to leave (e.g., Orlanski, 1976; Bennett, 1992). Stevens (1991) applied the Orlanski scheme to a GCM and obtained encouraging results; his approach was later taken up by Redler and Döscher (1997) in the CME framework.

Open boundary conditions have been a vexing issue in ocean modeling for two very distinct reasons. First, there is the fundamental mathematical problem of the wellposedness or otherwise of the underlying fluid-dynamical system. As Bennett (1992, Section 9.3) laid out in detail, the number of conditions that can and must be posed at an open boundary is dependent on the solution of the problem itself and hence not determined beforehand. In order to run a model forward with open boundaries, one would typically overspecify the boundary conditions, which could lead to internal inconsistencies, artificial fronts, and numerical instabilities. The numerical difficulties might be reduced by strong enough dissipation near the boundaries, with unclear consequences for the quality of the solution.

However, even if the mathematical foundation were established, there would be a second problem, namely the general lack of sufficient information about cross-boundary exchanges. Indeed, we feel that this "information problem" is the more severe one, since quite a number of GCMs have been run with open boundaries without displaying excessive numerical problems (e.g., Stevens, 1991; Redler and Döscher, 1997; C. Herbaut and J. Marshall, 1997, pers. comm., who ran a high-resolution Labrador Sea model with open boundaries).

Using an inverse approach addresses both fundamental problems of open boundary conditions: if observations are available within the model domain, the information contained therein could be propagated to the boundary, hence permitting one to infer what the boundary conditions ought to have been. In other words, the influence of the rest of the World Ocean on the modeled region-which in a regional model is exerted through the lateral boundary conditions - would be estimated from the observed response in the model interior. This aspect addresses the "information problem" of open boundaries. Moreover, if the mathematical ill-posedness leads to unphysical features of the solution, these can be penalized in the objective function employed in the inversion and hence eliminated. This recipe, while easily stated in principle, has hitherto been carried out only for relatively simple models, for example shallow-water models (e.g., Bennett and McIntosh, 1982; Bogden et al., 1996) or quasi-geostrophic models (e.g., Gunson and Malanotte-Rizzoli, $1996 \mathrm{a}, \mathrm{b})$. The reason is that the technical hurdles seemed too formidable to try this on a GCM since a nonlinear inverse approach would have to be combined with a GCM and the open boundary formulation. We report here what is, to the best of our knowledge, the first estimation of open boundary conditions using an ocean GCM. Because we are using a GCM, we can use heat and salinity conservation in the model interior as constraints for the advective fluxes of mass, heat, and salt across the lateral boundaries. 
This success has been made possible through R. Giering's recently developed software tool, termed the Tangent-Linear and Adjoint Model Compiler (TAMC, see Giering and Kaminski, 1998). Using the TAMC, we have constructed the adjoint to the GCM of Marshall et al. (1997a,b). We will refer to this model as the MIT GCM; details of the adjoint GCM development are described in Marotzke et al. (1999); an application to a global ocean estimation problem was discussed in Stammer et al. (1997). We then used the model version that incorporated open boundary conditions (C. Herbaut, 1997, pers. comm.), and generated the adjoint to the modified model with relative ease through successive applications of the TAMC.

The strategy described in this paper is, in principle, applicable to every regional modeling problem. Here, we apply it to the Indian Ocean configuration and annual mean data previously used by Lee and Marotzke (1997, LM97 hereinafter), where it promises to be particularly beneficial. LM97 highlighted a fundamental dichotomy in the views of how the meridional circulation of the Indian Ocean works. The analysis of hydrographic sections suggested that the zonally integrated deep mass inflow was moderate (10-15 Sv, $1 \mathrm{~Sv}$ is $10^{6} \mathrm{~m}^{3} / \mathrm{s}$, Macdonald and Wunsch, 1996; Robbins and Toole, 1997) or large (27 Sv, Toole and Warren, 1993) and presumably buoyancy-driven (Warren, 1994). In contrast, GCMs persistently did not produce significant deep inflow (Wacongne and Pacanowski, 1996; LM97; Garternicht and Schott, 1997). LM97 also found that meridional mass and heat transports were dominated by a wind-driven overturning cell that was confined to the thermocline. The analysis of a global high-resolution GCM (Garternicht and Schott, 1997) likewise showed a dominant shallow overturning cell.

These differing results underscore the large uncertainty in recent estimates of Indian Ocean meridional transports. On the one hand, one must question the representativeness of a single or very few hydrographic sections, the most densely sampled of which permits widely varying transport estimates from its temperature and salinity fields. Notice that Robbins and Toole (1997) quoted an uncertainty of $3 \mathrm{~Sv}$, which means that they obtained estimates with nonoverlapping error bars, depending on whether silica information was used or not. Hence, the silica and hydrography constraints either were incompatible with each other, or, more likely, the error bars were too optimistic.

The models, on the other hand, are consistent among themselves, but were not forced to consistency with climatological hydrography (Garternicht and Schott, 1997), or used unphysical sponge layers (LM97), or both (Wacongne and Pacanowski, 1996). Notice that model resolution and the presence or absence of a seasonal cycle did not have a large influence (Lee and Marotzke, 1998). Hence, we focus on the (arguably) weakest point of the LM97 model: we expand the inverse modeling framework to include the estimation of the open boundary conditions. We investigate whether the inflows and outflows now permitted, change the estimated structure and dynamics of the meridional overturning circulation. Moreover, we estimate the strength of the ITF and take a much closer look at the salinity budget, which received little attention from LM97. Finally, we define and analyze generalizations of meridional mass and heat transports appropriate in the presence 
of section-integrated mass transports, which is particularly important for the salt budget (Wijffels et al., 1992). So far, however, the problem has often been circumvented by only analyzing transports for the Indian and Pacific oceans together (e.g., Semtner and Chervin, 1992).

All models cited above used climatological hydrography for initialization, forcing, or as explicit constraints, and hence might all be negatively affected by the lack of spatial resolution in the climatology. This issue can be addressed by using the World Ocean Circulation Experiment (WOCE) Indian Ocean data set in GCM estimation studies. Work on this (the WOCE synthesis in the Indian Ocean) is ongoing, and its results will presumably supersede what is inferred about the circulation from the current study. Hence, this paper can be viewed as a sensitivity study, testing the impact of the open boundaries on the solution of LM97. Notice, however, that the WOCE expedition observed the Indian Ocean in a single year, whereas the climatology averages over many decades; differences in results must hence be expected.

This paper is organized as follows. Section 2 presents the data and models used. Section 3 investigates the impact of the open boundary conditions on a purely dynamical model run (without use of the adjoint). Section 4 analyzes our "best estimate" solution. Section 5 presents a number of sensitivity experiments. Discussion and conclusions follow in Section 6.

\section{Data and models}

a. Data

This study uses annually averaged climatological temperature and salinity from the Levitus World Ocean Atlas (Levitus and Boyer, 1994; Levitus et al., 1994). The wind stress is from Hellerman and Rosenstein (1983) and the surface heat and freshwater fluxes (precipitation minus evaporation, P-E) from Oberhuber (1988). All data sets have been interpolated onto the model grid for initialization, forcing, and comparison with the model estimates. The assumed uncertainties of the hydrographic data are plotted in Figure 1 and are the same as used earlier in Marotzke and Wunsch (1993) and LM97. The uncertainties of surface fluxes are assumed uniform in space, and chosen as $0.05 \mathrm{~N} / \mathrm{m}^{2}$ for wind stress, $50 \mathrm{~W} / \mathrm{m}^{2}$ for heat flux, and, in most experiments, $0.075 \mathrm{~m} / \mathrm{yr}$ for freshwater flux. This latter choice is far too optimistic and was kept due to an oversight. While we analyzed the model solution, its detrimental impact, particularly on the surface salinity field, became apparent. We, therefore, performed a sensitivity study with the more realistic assumed error of $0.5 \mathrm{~m} / \mathrm{yr}$, as in Schiller (1995).

\section{b. Model description}

The MIT Ocean GCM is based on the incompressible Navier-Stokes equations under the Boussinesq approximation, formulated in spherical geometry with height as vertical coordinate. In the version used here, the hydrostatic and rigid lid approximations are also 


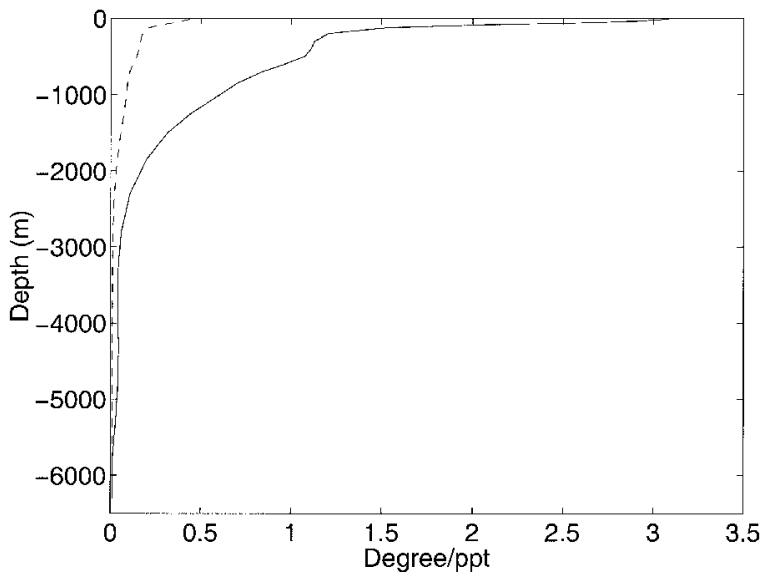

Figure 1. Prescribed standard errors for temperature (solid) and salinity (dashed). Units are degree and psu, respectively.

applied. The horizontal components of velocity, temperature, and salinity are solved prognostically, then vertical velocity and pressure are calculated diagnostically. A detailed description of the model formulation and numerical implementation is given in Marshall et al. (1997a, b).

The model domain represents the Indian Ocean north of 31S with the topography taken from the Gates and Nelson (1975) atlas (see LM97). The horizontal coordinates are spherical, and the resolution is $1.5^{\circ} \times 1.5^{\circ}$. Height is used as the vertical coordinate and there are 24 levels; the vertical interfaces of grid cells are placed at the depths of $25,50,75$, $100,150,250,350,450,550,650,750,950,1150,1350,1650,2050,2550,3050,3550$, $4050,4550,5050,5550$, and $6050 \mathrm{~m}$. Madagascar is submerged to $100 \mathrm{~m}$ depth. Open boundary conditions are applied to allow inflow and outflow to and from the Southern Ocean and the Indonesian Archipelago (details in the following section). The Red Sea and Persian Gulf have minor impact and are closed with 2-grid-cell wide sponge layers, in which temperature and salinity are relaxed to climatology on time scales of 1 and 5 days for the outer and inner grid points, respectively.

The timestep for the integration is $2 \mathrm{hrs}$. The eddy coefficients of subgrid scale mixing are chosen as $A_{h}=1.0 \times 10^{5} \mathrm{~m}^{2} / \mathrm{s}$ and $A_{v}=10^{-3} \mathrm{~m}^{2} / \mathrm{s}$ for horizontal and vertical viscosity, respectively, and $K_{h}=1.0 \times 10^{3} \mathrm{~m}^{2} / \mathrm{s}$ and $K_{v}=3 \times 10^{-5} \mathrm{~m}^{2} / \mathrm{s}$ for horizontal and vertical diffusivity, respectively. Convective adjustment of temperature and salinity is performed once per day if static instability arises.

\section{c. Open boundary conditions}

The implementation of the open boundary conditions into the MIT GCM is that of C. Herbaut (1997, pers. comm.). Open boundaries are placed at zonal velocity grid points at $118.5 \mathrm{E}$ and at meridional velocity grid points at $31 \mathrm{~S}$. The arrangement of grid cells near 


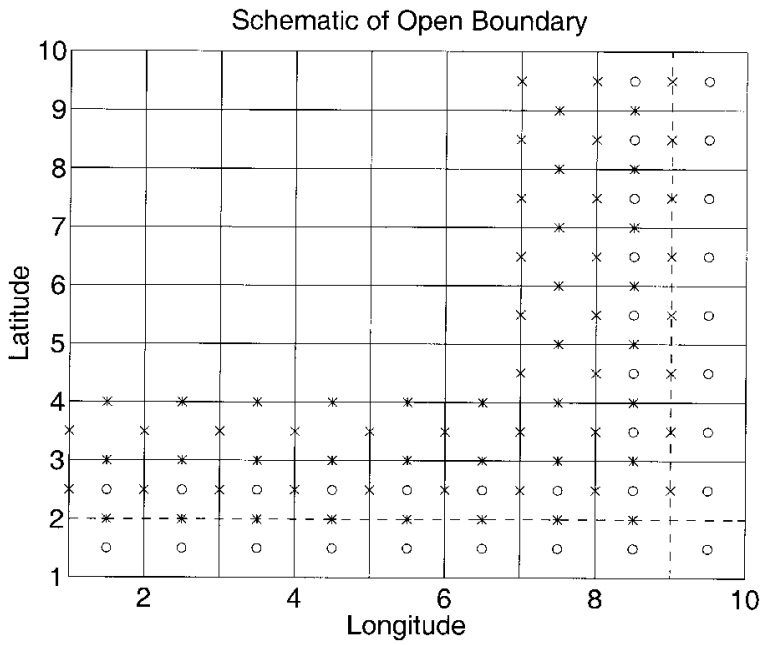

Figure 2. A schematic diagram of the open boundary condition. The dashed lines represent the positions of open boundaries. Circles represent temperature and salinity, x-marks zonal velocity, and stars meridional velocity. Temperature and salinity outside the open boundaries are prescribed, as are normal velocities at the open boundaries. At the interior grid points shown here, velocities are relaxed to prescribed values on a time scale of 1 day at those adjacent to the open boundary and 5 days at those away from the open boundary. All the prescribed values are taken first from a global model solution and then determined (estimated) by the optimization.

an open boundary is shown schematically in Figure 2. Normal velocities across the open boundary are prescribed in the prognostic model (and estimated in the inverse model). To guarantee mass conservation, we calculate the difference between the total inflow and outflow and subtract it evenly from all the grid points at the open boundaries. Model velocities (zonal and meridional) at the last and second last interior grid points are restored to prescribed values over time scales of 1 day and 5 days, respectively, for all vertical grid levels (again, in the inverse model these velocity values are estimated). Temperature and salinity are prescribed (or estimated) at all depths at the grid points just outside of the open boundaries. Heat and salt advection across the open boundaries is then handled by the standard centered-difference advection scheme of the model. This means that at outflow points, heat and salt are simply transported out of the model domain, whereas at inflow points, the prescribed exterior values are advected in. A newer version of the open boundary conditions uses upstream differencing across the open boundaries, avoiding the influence of the exterior fields at outflow points (C. Herbaut, 1998, pers. comm.), but this has not yet been implemented into the inverse model.

Notice that our open boundary conditions are computationally simpler than those of Stevens (1991), who calculated the normal velocity across the boundary from the linearized form of the equations of motion, then corrected it with plane wave velocity for tracer advections. For large-scale flows, this implies that the flow normal to the open 
boundary is almost in thermal-wind balance with the along-boundary density gradient. Previously, we had experimented with Stevens's boundary conditions in the Geophysical Fluid Dynamics Laboratory model; overall, it seems that the Herbaut formulation is more robust. This may be due to the smoothing that is performed dynamically when velocities are constrained (in the two grid cells adjacent to the boundary); temperature and salinity conspire so that the full equations of motion are maintained. In contrast, the calculation of velocities from prescribed density, as in the Stevens (1991) formulation, effectively introduces a differentiation which increases the noise level. The downside of the Herbaut formulation is that, for prognostic computations, complete velocity information is needed; we use the global ocean circulation estimates of Stammer et al. (1997).

The absolute velocity is prescribed (or restored) at all depths, at or near an open boundary, and hence no special provision must be made for the depth-averaged velocity. However, the calculation of the surface pressure in the presence of an open boundary requires some discussion. Generally, the surface pressure is calculated from the condition that the vertically integrated horizontal velocity is divergence-free (the rigid-lid approximation, see Marshall et al., 1997a,b for details). This condition leads to a Poisson equation for the surface pressure, as is shown by writing the horizontal momentum equation as

$$
\frac{\partial \mathbf{v}_{h}}{\partial t}=G_{v h}-\nabla_{h}\left(P_{S}+P_{H Y}\right)
$$

where $\mathbf{v}_{h}$ represents horizontal velocity and $G_{v h}$ represents advection, Coriolis, metric, gravitational, and forcing/dissipation terms in the zonal and meridional directions. The pressure field is separated into "surface pressure" $P_{S}$ and "hydrostatic pressure" $P_{H Y}$. The former is the pressure exerted by the fluid under the rigid lid at the surface, and is obtained from solving a Poisson equation (see below). The latter is defined in terms of the weight of water in a vertical column above the vertical coordinate $z$. Notice that both "surface" and "hydrostatic" pressures are hydrostatic in the model version used here, but we follow the standard oceanographic nomenclature (see Marshall et al., 1997b).

A Poisson equation for the surface pressure is obtained by integrating (1) vertically and taking its horizontal divergence, which gives

$$
\nabla_{h} \cdot\left(H \nabla_{h} P_{S}\right)=\nabla_{h} \cdot\left(H \overline{G_{v h}}\right)-\nabla_{h} \cdot\left(H \overline{\nabla_{h} P_{H Y}}\right)-\nabla_{h} \cdot \frac{\partial \hat{\mathbf{V}}}{\partial t} .
$$

The overbar represents the depth-mean, $H$ the ocean depth, and $\hat{\mathbf{V}}$ the vertically integrated horizontal velocity,

$$
\hat{\mathbf{V}}=\int_{-H}^{0} \mathbf{v d z}
$$

The surface pressure is found as a solution to (2); the appropriate boundary conditions are obtained as follows. By Gauss's theorem, (2) implies that, for arbitrary control areas with $\mathbf{n}$ 
denoting the unit normal vector,

$$
\mathbf{n} \cdot\left(H \nabla_{h} P_{S}\right)=\mathbf{n} \cdot\left(H \overline{G_{v h}}\right)-\mathbf{n} \cdot\left(H \overline{\nabla_{h} P_{H Y}}\right)-\mathbf{n} \cdot \frac{\partial \hat{\mathbf{V}}}{\partial t} .
$$

If the entire model area is chosen as the control area, $\mathbf{n}$ is the unit vector normal to the boundary. At a closed boundary, the kinematic boundary condition of no normal flow (implying $\partial \hat{\mathbf{V}} / \partial t=0$ ) automatically translates into the correct condition for the surface pressure gradient normal to the wall. At an open boundary, the normal flow is nonzero, but the terms on the right-hand side of (2) are still calculated such that the surface pressure gradient normal to the wall is consistent with the kinematic boundary condition. Hence, the open boundary requires only one addition to the model's Poisson solver, namely the third term on the right-hand side of (4).

We have stated which quantities are prescribed at the open boundaries of the prognostic model. The question of what values to choose will be taken up below; suffice it to say here that they will be determined as part of the solution of the inverse model, from observations and the model dynamics. We reiterate our point of view that it is the choice of these values, rather than the mathematical formulation of the open boundary conditions, that is the biggest problem in regional ocean modeling, thus motivating our elaborate procedure to estimate these values.

\section{d. Adjoint model and cost function}

A crucial tool in the task of fitting our model to the observations is the model's adjoint, which is an efficient way to calculate the sensitivity of one or more (but few) output variables to many input variables. Here, the input variables are a subset of the independent variables of the prognostic model, namely initial conditions in temperature and salinity, the surface fluxes (assumed time-independent), and the parameters of the open boundary conditions (temperature, salinity, and velocities, all taken time-independent). The output variable is a scalar "performance index," or cost function, expressing how well the model result fits the various constraints (see below). The result of the adjoint model, that is, the sensitivity of the cost function to changes in the independent parameters, provides crucial information about how to vary the latter efficiently in an optimization algorithm that minimizes the cost function. Here, the conjugate gradient method is chosen as the minimization algorithm (Press et al., 1992).

The adjoint of the MIT GCM is generated by applying the Tangent-linear and Adjoint Model Compiler (TAMC, Giering and Kaminski, 1998) to the GCM. The TAMC is a source translation code (FORTRAN-to-FORTRAN); it differentiates the code line by line and writes out the corresponding adjoint code. A detailed description of the steps involved in constructing the MIT adjoint model is given in Marotzke et al. (1999); the principles of adjoint code construction are outlined there, and are discussed comprehensively in Giering and Kaminski (1998). 
The cost function is defined as

$$
\begin{aligned}
J= & \sum\left(T_{N}-T_{d}\right)^{T} W_{d}^{T}\left(T_{N}-T_{d}\right)+\left(S_{N}-S_{d}\right)^{T} W_{d}^{S}\left(S_{N}-S_{d}\right) \\
& +\left(\vec{Q}-\vec{Q}_{d}\right)^{T} W_{d}^{\vec{Q}}\left(\vec{Q}-\vec{Q}_{d}\right) \\
& +\left(T_{N}-T_{0}\right)^{T} W_{s}^{T}\left(T_{N}-T_{0}\right)+\left(S_{N}-S_{0}\right)^{T} W_{s}^{S}\left(S_{N}-S_{0}\right),
\end{aligned}
$$

where $T, S$, and $\vec{Q}$ are the temperature, salinity and surface fluxes at ocean points, including those in the sponge layers and open boundaries. Subscripts $N$ and 0 represent the model values at timestep $n=N$ and 0 respectively, and subscript $d$ data value. The $W_{d}$ are the weight matrices for the various model/data misfits. They are diagonal (errors assumed uncorrelated) and taken as the reciprocal of the error variance for the hydrographic fields (Fig. 1) and 5 times of the reciprocal of the error variance of the surface fluxes. This latter choice reflects the assumption that, roughly, one surface flux estimate is worth as much as about 5 hydrographic data points. In other words, the number of degrees of freedom in one hydrographic profile is somewhat arbitrarily assumed to be about 5 , since one cannot count all (nominal) 24 observations at the vertical grid points as independent. The $W_{s}$ are the weight matrices for the temporal drift; they determine how severely deviations of the final model state from the initial state are penalized (Tziperman et al., 1992; Marotzke, 1992). This allows us to search for steady states without having to run the model to equilibrium for every single choice of control variables. As in LM97, we run the model over one year and choose $W_{s}$ as $W_{d} * 100$, implying that the adjustment time scale of the oceanic processes that we wish to be in equilibrium is 10 years or shorter (Marotzke, 1992). As the final step in defining the cost function, it is divided by the normalization factor of $N_{x} \times N_{y} \times 5 \times 2$, where $N_{x}$ and $N_{y}$ are the number of zonal and meridional grid points, respectively; 5 the assumed number of degrees of freedom in the vertical, and 2 the number of scalars (T, S). Hence, if the residuals making up the cost function each are of the order of one standard deviation, both the $T / S$-data and steadiness misfit terms should be of order one.

The inversion is started from a "prior estimate" or "first guess" for all independent parameters, among them those of the open boundary conditions. The "first guess" values for surface fluxes are taken directly from the climatologies. The "first guess" initial conditions for $T$ and $S$ are defined through a brief forward integration of the Indian Ocean model, starting from climatology (see below). All parameters of the open boundary conditions are interpolated from the $2^{\circ} \times 2^{\circ}$ global estimation from the TOPEX/ POSEIDON altimetry and surface observations of the year 1993, using the MIT GCM and its adjoint (Stammer et al., 1997). In the global estimate the Indonesian Archipelago is closed, owing to the smoothing in the topography, which makes the prior guess at the eastern boundary somewhat unsatisfactory. Notice, however, that due to the interpolation, the initial estimates of the open boundary conditions try to impose inflows of $3.67 \mathrm{~Sv}$ in the east and 3.24 Sv in the south. To conserve mass, the total net inflow of $6.91 \mathrm{~Sv}$ is subtracted evenly from all the grid points at the two open boundaries. This procedure results in a "first-guess" inflow of $2.96 \mathrm{~Sv}$ in the east and an outflow of equal amount in the south. 
Table 1. List of experiments.

\begin{tabular}{lllllc}
\multicolumn{1}{c}{ Expt. } & Init. cond. & Bound. cond. & Surf. forcing & Integ. type & Integ. length \\
Spin-up I & rest & open & fluxes + relax. & forward & 1 year \\
Spin-up II & rest & sponge & fluxes + relax. & forward & 1 year \\
Forward & rest & open & fluxes + relax. & forward & 100 years \\
Standard & Spin-up I & open & fluxes & optim. & 1 year \\
P-E & Spin-up I & open & fluxes & optim. & 1 year \\
Sponge & Spin-up II & sponge & fluxes & optim. & 1 year \\
Stability & Standard & open & fluxes & forward & 100 years \\
NOITF & P-E & open, no ITF & fluxes & forward & 60 years
\end{tabular}

Notice that we do not penalize deviations of the open boundary velocities from their prior estimates, formally giving them infinite freedom to adjust to whatever is demanded by the interior model and data. It turns out, though, that no large deviations arise. Notice further that the output of the adjoint calculation must be scaled for the descent algorithm, so that the optimization is performed in nondimensional space. The standard errors of surface fluxes, temperature, and salinity are used for the scaling of the gradients of the cost function with respect to these variables. The horizontal velocities at open boundaries are scaled uniformly, and arbitrarily, by $1 \mathrm{~cm} / \mathrm{s}$.

\section{Forward integration}

Prior to the optimization experiments, forward integrations of the GCM are performed in order to (1) see the difference the open boundary conditions make in our prognostic model, (2) find a dynamically balanced state to initialize the optimization experiments, (3) provide a reference point to assess the effect of the subsequent optimization experiments. Three forward integrations are performed; two are one year long and one is 100 years long.

The model ocean is initially at rest, and the hydrographic fields are taken as climatological values. Observed surface winds are used; the surface heat and freshwater fluxes are the sum of observations plus terms expressing relaxation on a timescale of 1 month to observed sea surface temperature (SST) and sea surface salinity (SSS). The Persian Gulf and Red Sea are closed with sponge layers in all experiments, while either sponge layers (with restoring towards Levitus temperature and salinity, as in LM97) or open boundaries are applied to the Indonesian Archipelago and the southern boundary. The one year integration with open boundary conditions is referred to as Spin-up I, while that with sponge layers is referred to as Spin-up II (Table 1).

The meridional overturning streamfunction, which is defined as vertical integration of meridional velocity from the bottom upward, is shown in Figure 3. Notice that in the presence of the mass influx from the ITF this quantity is no longer rigorously defined at the ITF latitudes. As the zonally and vertically integrated meridional velocity still gives the best impression of the basin-wide overturning circulation, we decided to start the integration from the bottom and simply accept that the values are nonzero at the surface. 


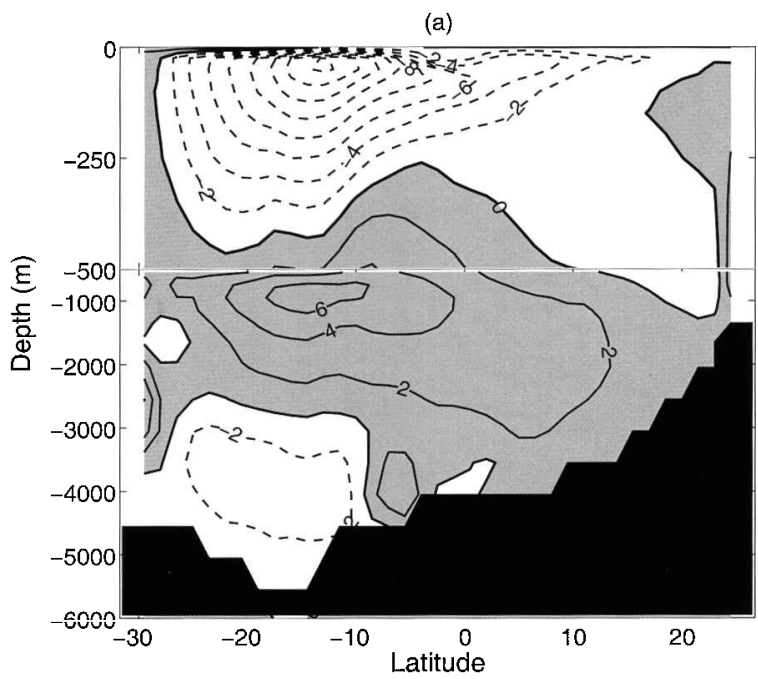

(b)

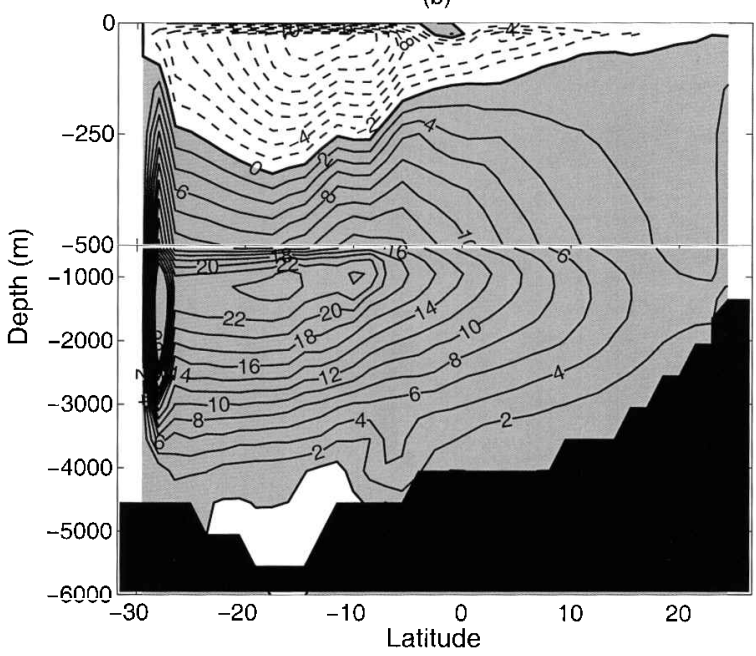

Figure 3. Meridional streamfunction of (a) Spin-up I, and (b) Spin-up II. Contour interval 2 Sv; shaded areas and solid lines indicate positive values, dashed lines negative values, thick lines represent $0 \mathrm{~Sv}$. The circulation is such that high values are to the right (clockwise around a high).

Notice that the surface values would have been zero without any mass source at the boundary. Previously, Garternicht and Schott (1997) have likewise used this remedy.

The meridional overturning streamfunctions of both spin-ups show a counterclockwise cell near the surface, of strength $16 \mathrm{~Sv}$ but reaching a greater depth in Spin-up I than in Spin-up II. Below this thermocline meridional cell lies a clockwise cell, which has a magnitude of $6 \mathrm{~Sv}$ maximum in Spin-up I and a much larger value of $24 \mathrm{~Sv}$ in Spin-up II. There is a third clockwise cell at the bottom in Spin-up I, which is absent in Spin-up II. The 


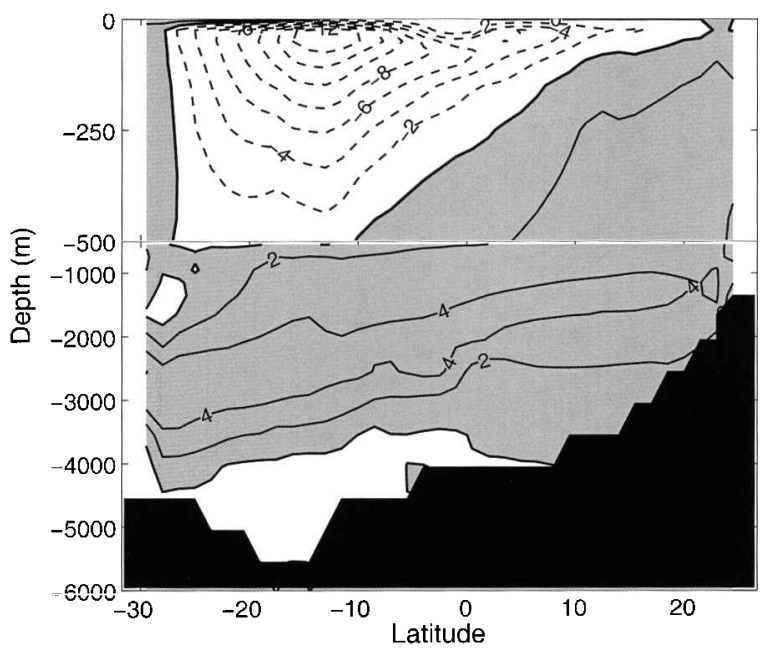

Figure 4. Meridional overturning streamfunction of Forward Expt. Contour interval 2 Sv; shaded areas and solid lines indicate positive values, dashed lines negative values, thick lines represent $0 \mathrm{~Sv}$. The circulation is such that high values are to the right (clockwise around a high).

vigorous inflow at mid-depth was seen in the purely prognostic model solutions of Wacongne and Pacanowski (1996) and LM97, and shown by the latter to be an artifact of the southern sponge conditions.

The integration of Spin-up I is continued for a total of 100 years, and is referred to as Forward Expt. (Table 1). The meridional overturning at year 100 is plotted in Figure 4. Compared to Spin-up I, one observes that although the thermocline cell remains almost unchanged, the subsurface cell has weakened by $2 \mathrm{~Sv}$. We note that the spinup time of the meridional overturning is surprisingly short, similar to the closed-domain experiments of LM97.

\section{Standard Experiment}

The Standard Experiment is initialized with the 1-year spin-up using open boundary conditions in the east and south (Spin-up I) and optimized by the forward and adjoint models with open boundary conditions (Table 1). In this section, we will focus on those results of this experiment that are most strongly affected by the open boundaries (in comparison with LM97).

\section{a. Search for a minimum}

For our choice of weight matrices, the magnitude of each of the two major contributors to the cost function (T/S data misfit, $T / S$ steadiness misfit) should be of order one. Cost and norm of cost gradient, as a function of conjugate gradient iteration, are plotted in Figure 5. The cost value decreases quickly during the first few iterations and then more slowly, 
(a)

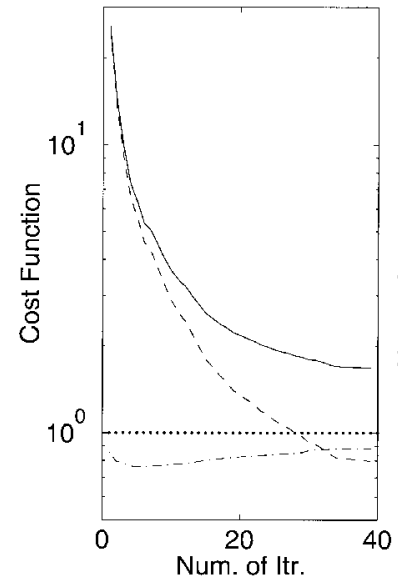

(b)

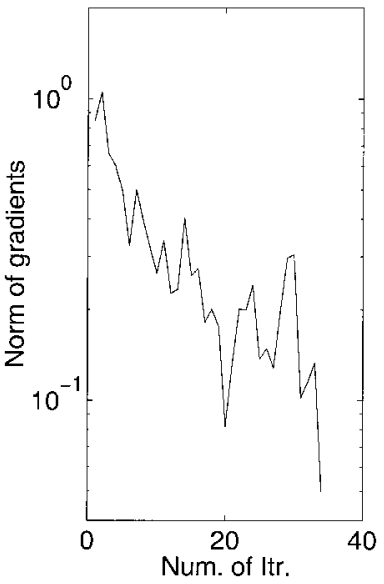

Figure 5. (a) Normalized cost function; the solid, dashed, and dash-dotted lines represent total cost, temporal drift, and model-data misfit, respectively. The dotted line represents 1 standard deviation rms deviation in each of temporal drift and model-data misfit. (b) Normalized local downhill gradient.

reaching the noise level (a value of 2) at about 40 iterations. The temporal drift contribution decreases monotonically with iteration number, but the model/data misfit shows a small increase after an initial reduction. Notice that Figure 5a is semilogarithmic, so the leveling off of total and steadiness cost means faster than exponential approach to a minimum. The local downhill gradient generally decreases with iteration count for the first 30 iterations and then shows oscillations toward the end of the experiment, when the downhill direction is not clear anymore as the cost function approaches a minimum. We conclude that a local minimum has been found, which additionally has a cost value that, on average, shows consistency of all constraints.

\section{b. Circulation and boundary currents}

The estimated horizontal velocity is plotted in Figure 6 for levels 1 (12.5 m depth) and 4 $(87.5 \mathrm{~m})$. The wind-driven surface currents are readily identified, like the westward South Equatorial Current between 10S and 20S, the eastward Equatorial Countercurrent near the equator, and the Somali Current and the anticyclonic Arabian Sea gyre. Compared to the closed-domain results of LM97, the estimated velocity field shows a marked improvement near the southern boundary, with a reasonably strong (for a model of this resolution) Agulhas Current leaving the model domain. Its maximum southward velocity is $13 \mathrm{~cm} / \mathrm{s}$, and its southward baroclinic transport (defined as the integral over all contiguous southward motion in the Agulhas region) is $42 \mathrm{~Sv}$. There is a much stronger East Madagascar Current ( $5 \mathrm{~cm} / \mathrm{s}$ maximum southward velocity, $28 \mathrm{~Sv}$ baroclinic transport) than in LM97, and significant flow (13 Sv) southward through the Mozambique Channel, again in contrast to LM97. Moreover, there is a shallow (depth less than $100 \mathrm{~m}$ ) Leeuwin 
(a)

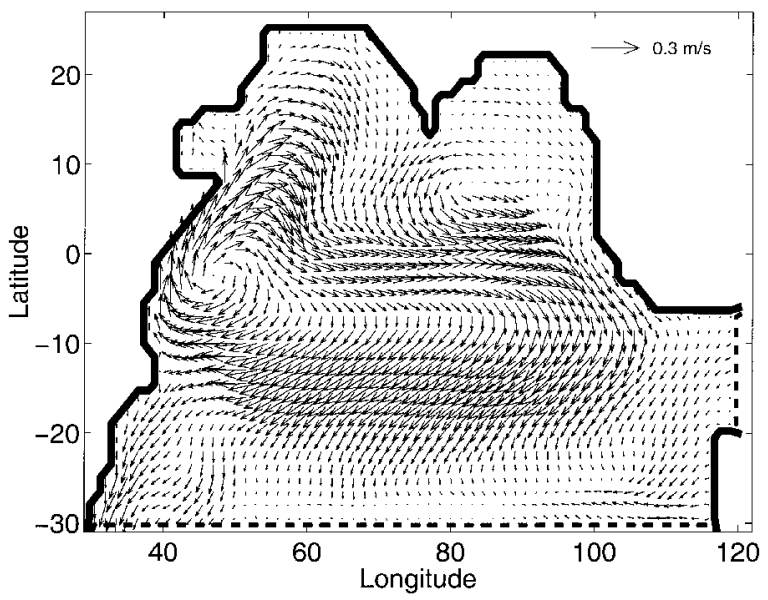

(b)

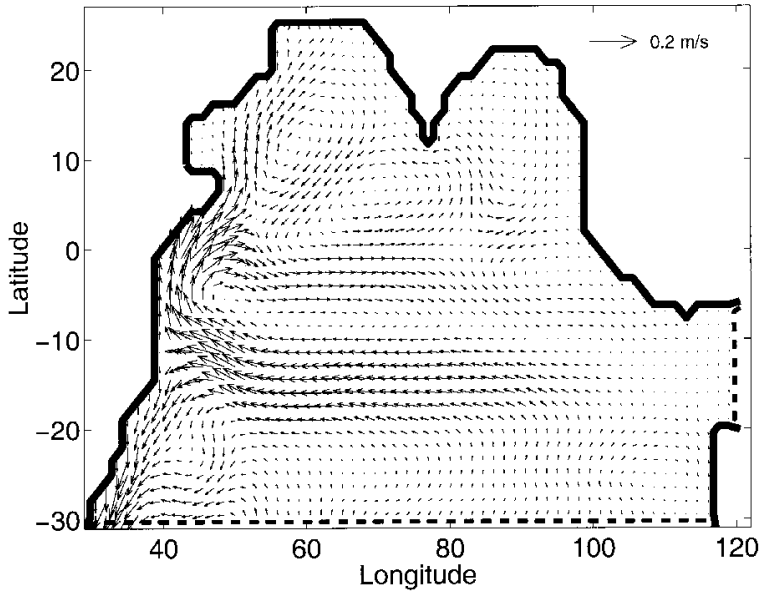

Figure 6. Horizontal velocity at the depth of (a) $12.5 \mathrm{~m}$ and (b) $87.5 \mathrm{~m}$. Scale as indicated by reference arrows.

Current of $0.5 \mathrm{~Sv}$ southward and a clearly recognizable, albeit weak, inflow from the Indonesian Passages.

Meridional velocity at $28 \mathrm{~S}$ is displayed in Figure 7, showing that the Agulhas Current extends downward to a depth of 1,800 m, and the continuation of the East Madagascar Current extends deeper still. Figure $7 \mathrm{~b}$ shows the changes in the target meridional velocity, compared to the first estimate, at 28S. Apart from a strengthening of the Leeuwin Current, the induced changes are concentrated below $1000 \mathrm{~m}$ depth, with values often greater than $1 \mathrm{~cm} / \mathrm{s}$. There is a tendency toward increased outflow near the western boundary and generally above the bottom, where the strongest changes occur, but there does not emerge a 
(a)

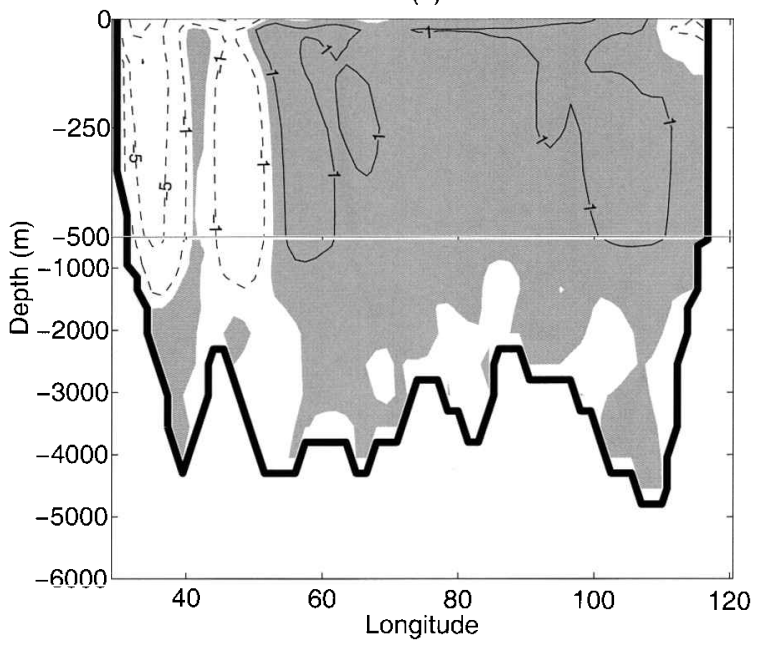

(b)

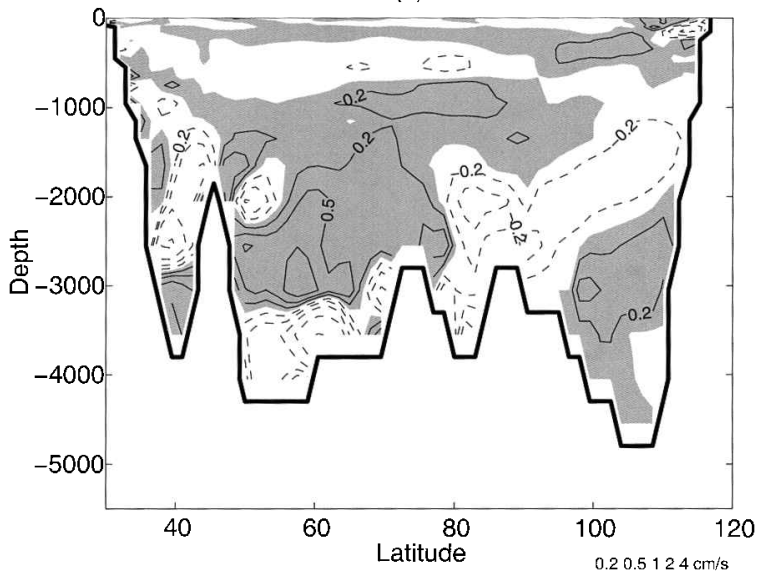

Figure 7. Zonal sections of meridional velocity field at 28S (inside the boundary layer). Shaded areas represent northward velocity. (a) Model estimate; contour intervals are 1, 5, and $10 \mathrm{~cm} / \mathrm{s}$. (b) Deviation of estimated target velocity from first guess; contour intervals are $0.2,0.5,1,2$, and $4 \mathrm{~cm} / \mathrm{s}$.

clear tendency. At this point, we can say that the open boundary estimation does change the flow across the boundary, without however identifying a clear cause.

\section{c. Indonesian throughflow}

Meridional sections of zonal velocity and salinity across the North Australian Basin, near the open eastern boundary, are plotted in Figure 8 and Figure 9, respectively. The surface current is westward from the Pacific into the Indian Ocean, with a maximum of 

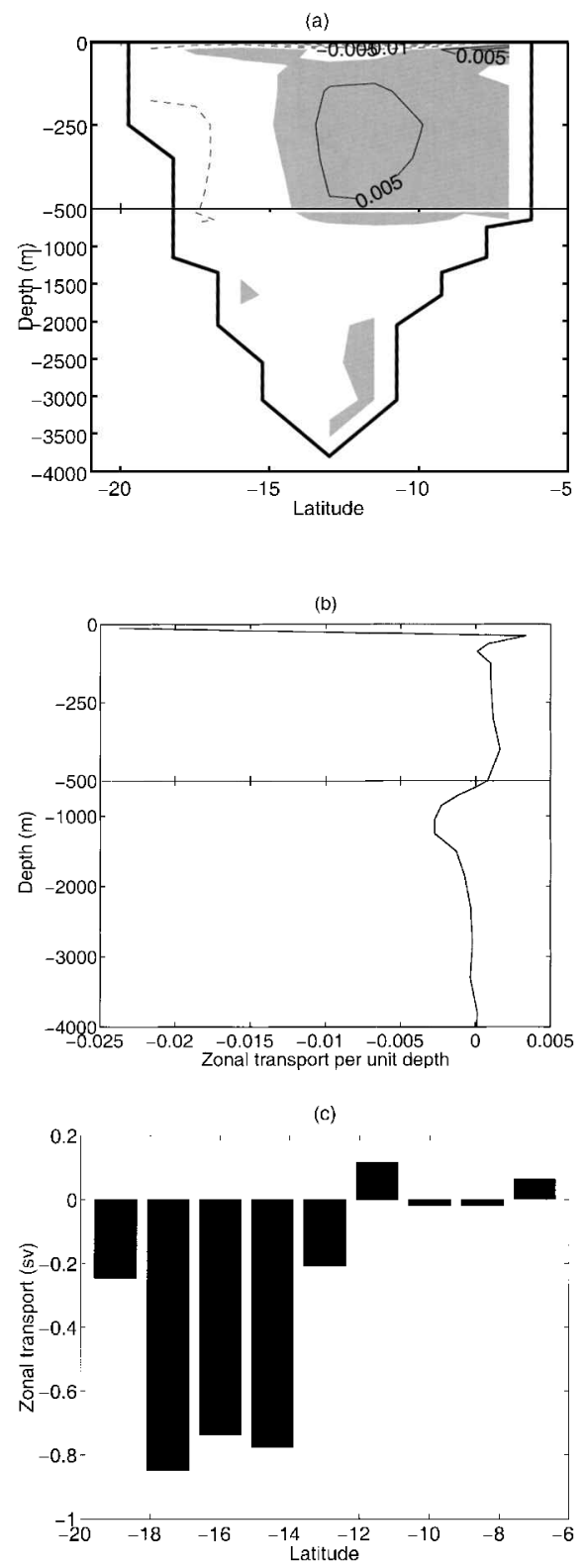

Figure 8. (a) Meridional section of zonal velocity at $118 \mathrm{E}$, contour interval $0.005 \mathrm{~m} / \mathrm{s}$, (b) vertical distribution of meridionally integrated zonal transport per unit depth across $118 \mathrm{E}$, in Sv/m, and (c) vertically integrated zonal transport across $118 \mathrm{E}$ as a function of latitude, in Sv. 
(a)

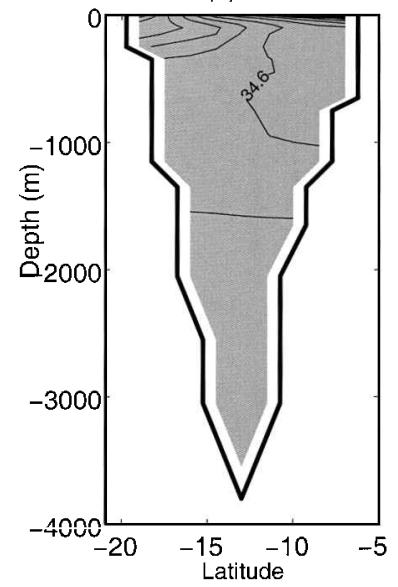

(b)

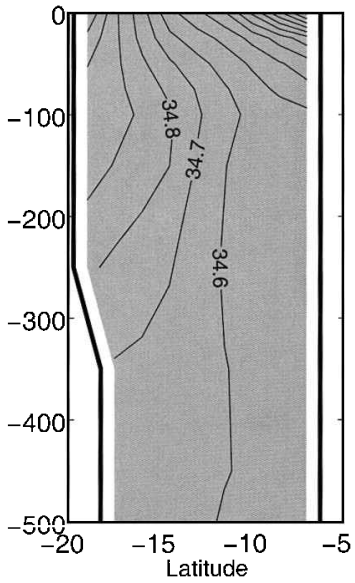

Figure 9. Meridional sections of salinity at 118E. (a) Full depth, (b) enlargement of upper $500 \mathrm{~m}$. Contour interval is $0.1 \mathrm{psu}$.

$3.1 \mathrm{~cm} / \mathrm{s}$ (Fig. 8). Below the surface, a very shallow South Java Current flows eastward at the northern end of the section. Below, down to 500-1000 m, there lie two alternating currents of about $0.5 \mathrm{~cm} / \mathrm{s}$, an eastward one in the northern half of the basin and a westward one in the southern half, the latter with its maximum leaning on the southern boundary. Meridionally averaged zonal transport across the basin is shown in Figure 8b; beneath the very shallow westward transport, there is eastward transport down to about $500 \mathrm{~m}$, with westward transport underneath. This picture shares some features with the geostrophic transport estimates of Fieux et al. (1996) for February 1992 (their Fig. 2a); they likewise found mid-depth eastward transport, which was centered around $500 \mathrm{~m}$, however. Depending on their choice of reference level, they also had weak westward transport at depth. For February 1992, Fieux et al. (1996) had eastward flow in the top $100 \mathrm{~m}$ and maximum westward transport beneath, in contrast to our surface-only westward flow. For August 1989, however, they inferred maximum westward transport right at the surface, and given the overall very large temporal variability, our solution is consistent with the geostrophic estimates. The total westward transport across the basin is $2.7 \mathrm{~Sv}$ and is largely confined to the upper 2,000 $\mathrm{m}$ (Fig. 8b). This value is near the low end of the range of previous estimates (Godfrey, 1996) but is perfectly consistent with them.

The vertically integrated zonal transports across the North Australian Basin are shown in Figure $8 \mathrm{c}$. The maximum eastward transport occurs at $11.5 \mathrm{~S}$, the maximum westward transport at 17.5S. Compared to a synoptic section (Fig. 3 of Fieux et al., 1996), we completely lack the eddy signal, but it is clear that strong horizontal recirculation is likely to occur. Notice, however, that our picture of horizontal "gyre" flow is entirely opposite to that of Meyers et al. (1995, their Fig. 3), who from a 6-year average of expendable bathythermograph (XBT) sections estimate eastward transports (relative to $400 \mathrm{~m}$ ) in the 
southern portion and westward transports in the northern part. Figure 9 shows that there is a salinity front at around $12 \mathrm{~S}$, reaching to $1000 \mathrm{~m}$, which appears to dominate the thermal wind shear in our solution. This front in our solution is consistent with the synoptic section of Fieux et al., (1994, their Fig. 3b), but would be hard to capture with the XBT network, especially since there is no clear $T / S$ relationship (Fieux et al., 1994, their Figs. 3a and 3b). Hence, it seems unlikely that the Meyers et al. (1995) circulation scheme is correct, but presumably clarification will come from the WOCE synthesis only.

\section{d. Near-surface salinity}

We will here concentrate on the estimated near-surface salinity field because it was not discussed by LM97 and because one would expect a significant impact of the ITF on the salinity, owing to the import of low-salinity Pacific water (e.g., Bray et al., 1997). In contrast to LM97, we can properly address this issue, at least within the framework of an annual-mean climatology.

Estimated and observed sea surface salinity (SSS) and their difference are shown in Figure 10. The deviation from steady state is small and not shown here. The pronounced SSS maximum near the Gulf of Aden is weaker in the estimate, indicating too weak lateral salt forcing. The local minimum in the northwestern Bay of Bengal is not reproduced, possibly because the runoff from the Ganges and Brahmaputra rivers is not included here, but also because we cannot reproduce the westward current along the northern boundary, which shows a pronounced seasonal cycle (e.g., Lee and Marotzke, 1998). A perhaps more interesting discrepancy arises in the southeastern Arabian Sea, where the front separating salty western and fresh eastern waters is much sharper in the model estimate and runs farther to the west. It appears that the model has reinstated sharp gradients that were smoothed during the construction of the climatology. The WOCE section I1 ran too far north to help decide whether the model estimate or the climatology is more credible (it cut the front where there is agreement), and section I2 ran too far south. But the Wyrtki (1988) atlas positions the surface front more in agreement with our results; moreover, Wyrtki (1988) shows two distinct fronts at $100 \mathrm{~m}$ depth, just like in our estimate (figure not shown).

Perhaps the most interesting discrepancy arises in the eastern equatorial region, where in the climatology the SSS front intersects the coastline around the equator, while model currents transport low-salinity water far into the southern hemisphere, clearly feeding the low-salinity tongue in the southeastern Indian Ocean (compare Figs. 10a and 6a). Here, the Wyrtki (1988) atlas appears more consistent with Levitus, although the data coverage does not allow firm conclusions; in contrast, the WOCE section I2 shows stronger gradients between $90 \mathrm{E}$ and 100E (figure not shown).

One disturbing feature of our model is the tiny deviation of estimated P-E from climatology (less than $0.001 \mathrm{~m} / \mathrm{yr}$, figure not shown), in spite of large SSS residuals, which should induce larger P-E corrections. It was the closer inspection of this contradiction which led us to recognize the erroneous P-E standard error (notice that LM97 used an error 
(a)

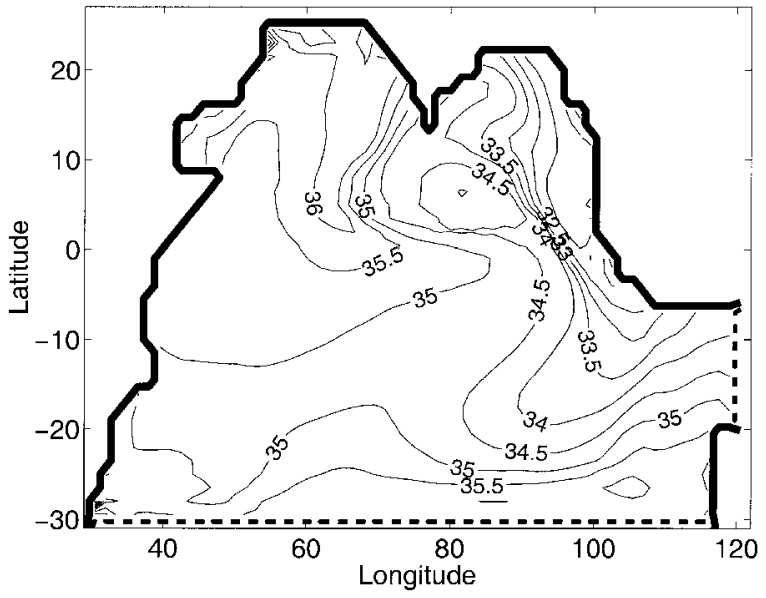

(b)

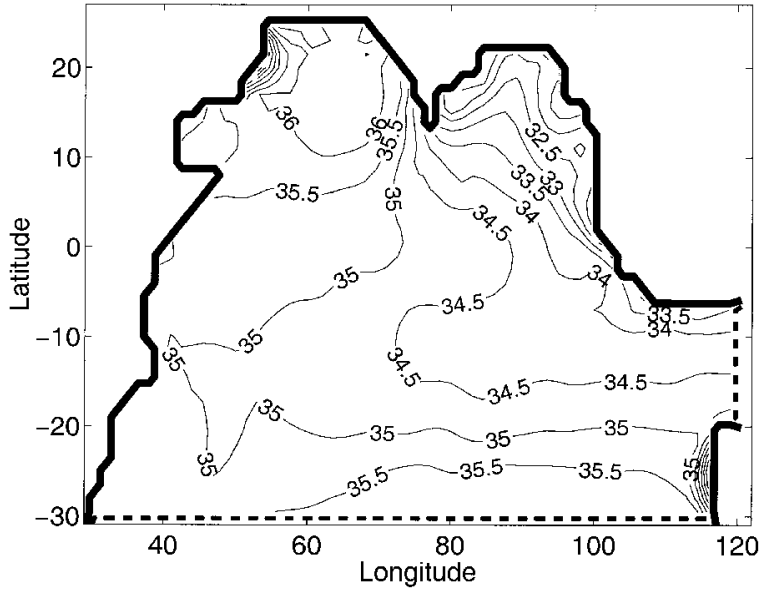

(c)

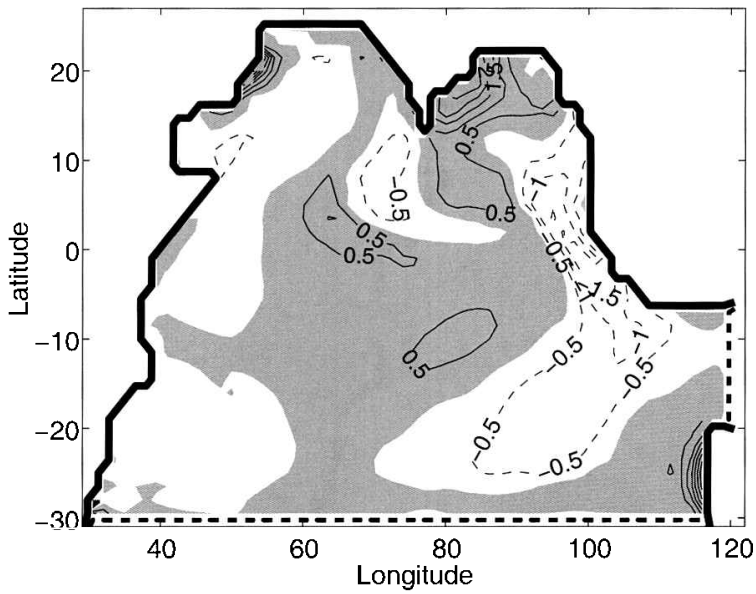

Figure 10. (a) Estimated salinity, (b) observed salinity, (c) model/data misfit, at the depth of $12.5 \mathrm{~m}$. Contour interval is $0.5 \mathrm{psu}$. 


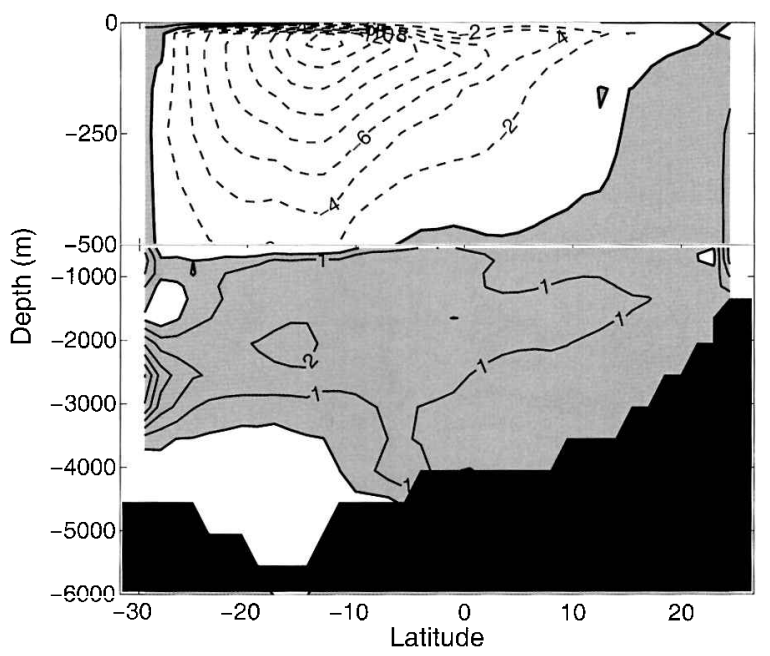

Figure 11. Meridional streamfunction of Standard Expt. Shaded areas and solid lines indicate positive values, contour interval $1 \mathrm{~Sv}$; dashed lines negative values, contour interval $2 \mathrm{~Sv}$; thick lines represent $0 \mathrm{~Sv}$. The circulation is such that high values are to the right (clockwise around a high).

of $0.075 \mathrm{~m} / \mathrm{month}$, not $0.075 \mathrm{~m} / \mathrm{yr}$, as incorrectly stated in their paper). We repeated the experiment with an assumed P-E error of $0.5 \mathrm{~m} / \mathrm{yr}$ (Expt. P-E, Table 1). While indeed the estimated P-E deviates more from the climatology, by up to $0.4 \mathrm{~m} / \mathrm{yr}$, and the SSS residuals are reduced somewhat, no qualitative change takes place, and subsurface results are nearly identical.

\section{e. Meridional overturning}

The zonally integrated meridional overturning streamfunction is plotted in Figure 11. A dominant counterclockwise cell is present in the upper $700 \mathrm{~m}$. Its magnitude is $16 \mathrm{~Sv}$ and the maximum is located at $12 \mathrm{~S}$ and about $50 \mathrm{~m}$ depth. The northward flowing water upwells continuously north of $12 \mathrm{~S}$, then returns in the wind-driven surface Ekman layer. We have confirmed that this overturning cell is indeed wind-driven, by separately turning off wind and surface buoyancy forcing of the optimized solution (following LM97). The shallow overturning carries equatorial surface water of high temperature southward and dominates the meridional heat transport; it is similar both in magnitude and depth with that estimated by LM97, who used a closed basin. Hence, we conclude that it was not the presence of closed boundaries that led to the differences between LM97 and Toole and Warren (1993). Notice that our results are also quite similar to the analysis of Garternicht and Schott (1997), except north of the equator where our overturning is considerably stronger. Below $500 \mathrm{~m}$ depth, there is a clockwise cell of $2 \mathrm{~Sv}$, similar in structure but weaker than in the forward experiment (compare with Fig. 4). 


\section{f. Meridional transports of heat and freshwater}

In general, a meridional "temperature transport" across an arbitrary zonal section can be calculated from a model solution as

$$
Q(y) \equiv c_{p} \rho_{0} \iint \nu T d x d z,
$$

where $c_{p}$ is specific heat, $\rho_{0}$ reference density, and $v$ and $T$ are meridional velocity and temperature, respectively. Notice that we do not call this a "heat transport," a term often reserved for mass-balanced flow; i.e., where the velocity integral over a section vanishes. This is not the case here, due to the net inflow of mass at the Indonesian Archipelago. In analyzing heat and freshwater fluxes, we thus face a conceptual difficulty related to the one with the streamfunction. In particular, the temperature transport depends on the zero point of the temperature scale, while the divergent part of the freshwater flux would be swamped by the net mass transport (most of which is freshwater, see the discussion in Wijffels et al., 1992). Typically, one chooses some sensible reference temperature and salinity, against which model $T$ and $S$ are evaluated.

In the following, we present a procedure which will give meaningful definitions of heat and freshwater transports in the presence of mass sources, while allowing us to evaluate the contributions of the mass source to the heat and salt budgets, and naturally suggesting choices for the reference values. We do not present a unique solution but a generally applicable procedure for models with open boundaries and for segments of multiply connected domains (like the Indian Ocean portion of a global model). The discussion is for temperature; the treatment of salinity is completely analogous.

First, we subtract from the temperature transport the cumulative contribution by the ITF, $Q_{I T F}(y)$, at each latitude,

$$
\begin{aligned}
\tilde{Q}(y) & \equiv Q(y)-c_{p} \rho_{0} \int_{y}^{Y_{N}} \int_{-H(y)}^{0} u T d z d y \\
& \equiv Q(y)-Q_{I T F}(y),
\end{aligned}
$$

where $Y_{N}$ is the northern edge of the throughflow and $H(y)$ is the ocean depth. Next, we decompose $Q_{I T F}(y)$ by defining a cumulative mass flux,

$$
M(y) \equiv \int_{y}^{Y_{N}} \int_{-H}^{0} u d z d y,
$$

and a section mean temperature,

$$
T_{I T F} \equiv \frac{1}{A} \int_{Y_{S}}^{Y_{N}} \int_{-H}^{0} T d z d y,
$$


where $A$ is the area of the boundary section and $Y_{S}$ the southern edge of the throughflow, to obtain

$$
\begin{aligned}
Q_{I T F}(y) & =c_{p} \rho_{0} \int_{y}^{Y_{N}} \int_{-H(y)}^{0} u\left(T_{I T F}+T^{\prime}\right) d z d y \\
& \equiv c_{p} \rho_{0} M(y) T_{I T F}+\tilde{Q}_{I T F}(y),
\end{aligned}
$$

where $T^{\prime}$ is the deviation of temperature from $T_{I T F}$ and $\tilde{Q}_{I T F}(y)$ is the ITF temperature transport relative to the ITF section mean temperature. Notice that $u$ rather than $u^{\prime}$ must be used in (10) since the lower limit of the integral is not $Y_{S}$ but variable. With (10), (7) becomes

$$
\tilde{Q}(y)=Q(y)-c_{p} \rho_{0} M(y) T_{I T F}-\tilde{Q}_{I T F}(y) .
$$

On the other hand, the total temperature transport across a zonal section, $Q(y)$, can be decomposed according to

$$
\begin{aligned}
Q(y) & =c_{p} \rho_{0} \boldsymbol{\int} \boldsymbol{\int} v d x d z \cdot \frac{\boldsymbol{\int} \boldsymbol{\int} T d x d z}{\boldsymbol{\int} \boldsymbol{\int} d x d z}+c_{p} \rho_{0} \int \boldsymbol{\int} v^{\prime} T^{\prime} d x d z \\
& \equiv c_{p} \rho_{0} M(y) \bar{T}(y)+Q^{\prime}(y),
\end{aligned}
$$

where overbar and prime on $T$ and $v$ represent zonal section mean and deviation from the section mean, respectively. The net cumulative inflow $M(y)$, from the Indonesian Archipelago north of $y$, has to flow southward in order to conserve mass. The first and second terms on the right-hand side of (12) represent temperature transport by the net mass transport and by the mass-compensated flow (heat transport proper), respectively. When the two decompositions for $Q(y),(11)$ and (12), are equated,

$$
c_{p} \rho_{0} M(y) \bar{T}(y)+Q^{\prime}(y)=\tilde{Q}(y)+\tilde{Q}_{I T F}(y)+c_{p} \rho_{0} M(y) \cdot T_{I T F},
$$

it follows that

$$
Q_{d i v}(y) \equiv \tilde{Q}(y)+\tilde{Q}_{I T F}(y)=Q^{\prime}(y)+c_{p} \rho_{0} M(y)\left[\bar{T}(y)-T_{I T F}\right] .
$$

Eq. (14) offers two complementary interpretations of ocean heat transport. Both sides of the equation represent the divergent part of the temperature transport and in steady state are balanced by the integral over the surface heat flux. The left-hand side is the sum of the "interior" heat transport and the portion of ITF temperature transport relative to the ITF section mean temperature. We are thus able to read off the "heating" or "freshening" by the ITF, without being troubled by mass imbalances or concomitant questions of temperature or salinity scale. Equally valid, the right-hand side says that the total divergent heat transport is due to the mass-balanced part of the flow, plus the change in section-mean temperature that the cumulative mass flux experiences. Again, in steady state the surface heating would be distributed over these two processes. 


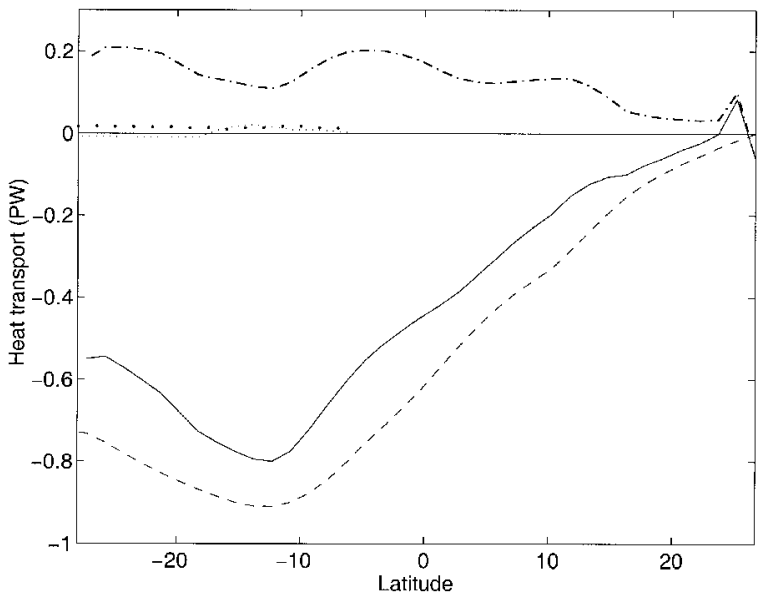

Figure 12. Meridional heat transport. The solid line represents the divergent component of temperature transport, with the contribution from the ITF included, i.e., $Q_{d i v}(y)$ from Eq. (14). The dashed line represents the zonally and meridionally integrated surface heat flux. The dash-dotted line is the solid line subtracted by the dashed line, i.e., the residual owing to changes in heat content. The thin dotted line represents the "ITF heating," $\tilde{Q}_{I T F}(y)$ of (10). The heavy dotted line represents the warming of the net mass flux relative to the ITF section mean temperature, i.e., the term $M(y)$ $\left[\bar{T}(y)-T_{I T F}\right]$ of (14).

The choice of $T_{I T F}$ as a reference temperature is not unique, but our generalized definition of heat transport has the advantage that it is continuously applicable, since at any given latitude the mass influx is taken into account. Not surprisingly, we find this particularly beneficial for the estimated salinity transport. Notice that this is converted into implied surface freshwater flux by dividing by a reference salinity of 35 .

The divergent heat transport $Q_{d i v}(y)$, with a finite value in the northern sponge layer removed, is plotted in Figure 12; it shows a maximum of $0.8 \mathrm{PW}$ southward, at $12 \mathrm{~S}$, slightly larger than LM97. Also shown in Figure 12 is the integral, starting from the northern boundary, of the estimated surface heat flux (dashed), and the difference between divergent ocean transport and integrated surface flux (dash-dotted); the latter reflects deviations from a steady state. Overall, we see that the ocean transport is somewhat weaker than what is demanded by the surface flux.

Figure 12 also shows the "ITF heating" term $\tilde{Q}_{I T F}(y)$ (thin dots) and the warming of the net mass flux, $c_{p} \rho_{0} M(y)\left[\bar{T}(y)-T_{I T F}\right]$ (heavy dots). Both terms are very small, in fact smaller than the residual; while the two are not independent of each other, it is fair to say that the ITF in our model does not contribute significantly to the Indian Ocean heat balance, in contrast to widespread expectation(Godfrey, 1996). In particular, the ITF effect cannot explain the greater deviation between ocean transport and surface flux in the ITF region; we have been unable to find a clear cause for the increased unsteadiness there.

Divergent model freshwater transport and the surface flux are reasonably well balanced between the northern sponge layer and the equator, as evidenced by the flat residual curve 


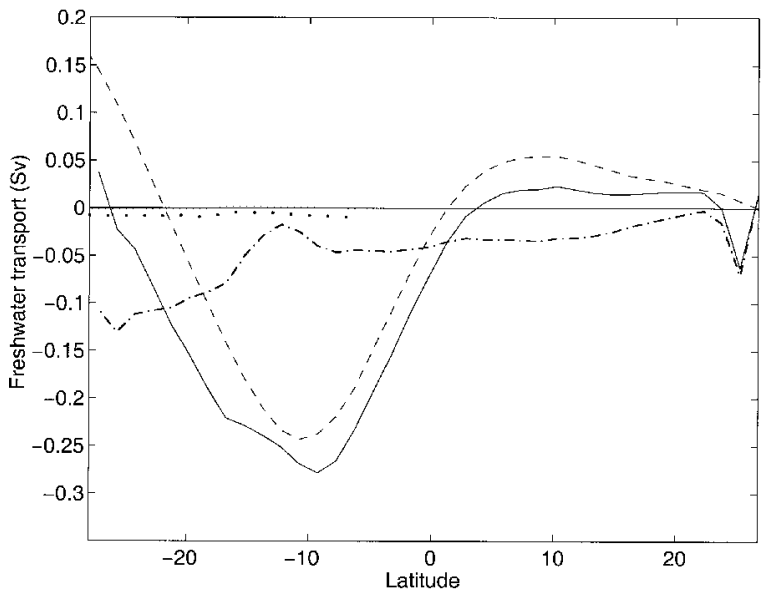

Figure 13. Meridional freshwater transport. The solid line represents the divergent component of freshwater transport, with the contribution from the ITF included, cf. $Q_{d i v}(y)$ from Eq. (14). The dashed line represents the zonally and meridionally integrated surface freshwater flux. The dash-dotted line is the solid line subtracted by the dashed line, i.e., the residual owing to changes in freshwater (or salt) content. The thin dotted line represents the "ITF freshening," cf., $\tilde{Q}_{I T F}(y)$ of (10). The heavy dotted line represents the freshwater transport of the net mass flux relative to the ITF section mean salinity, cf., the term $M(y)\left[\bar{T}(y)-T_{I T F}\right]$ of (14).

in Figure 13. Freshwater transport is weak in the northern Indian Ocean, reflecting the large differences in P-E between the evaporative Arabian Sea and the large precipitation over the Bay of Bengal. Between the equator and about 10S, a southward freshwater transport reaches $0.29 \mathrm{~Sv}$, which is reduced to about zero by the strong evaporation over the southern subtropics. As is the case for the heat budget, the ITF plays a negligible role in the freshwater budget, again counter to widespread expectation but consistent with Macdonald and Wunsch (1996) who found their global linear inverse model to be relatively insensitive to widely varying assumptions about ITF strength. Notice that our estimated freshwater transport (and hence the integrated Oberhuber, 1988, transport estimate) bears little resemblance to the one by Wijffels et al. (1992), which is based on the Baumgartner and Reichel (1975) compilation. In particular, Wijffels et al. (1992) conclude that the Indian Ocean transports freshwater northward at almost all latitudes. While it is unclear how our model would have dealt with the Baumgartner and Reichel (1975) climatology, this again points at the enormous uncertainty in P-E over the ocean.

\section{Sensitivity and stability experiments}

In this section, we perform two optimization experiments as sensitivity tests, and four forward integrations using optimized initial conditions and surface forcing in order to test the stability of the optimized steady state. 


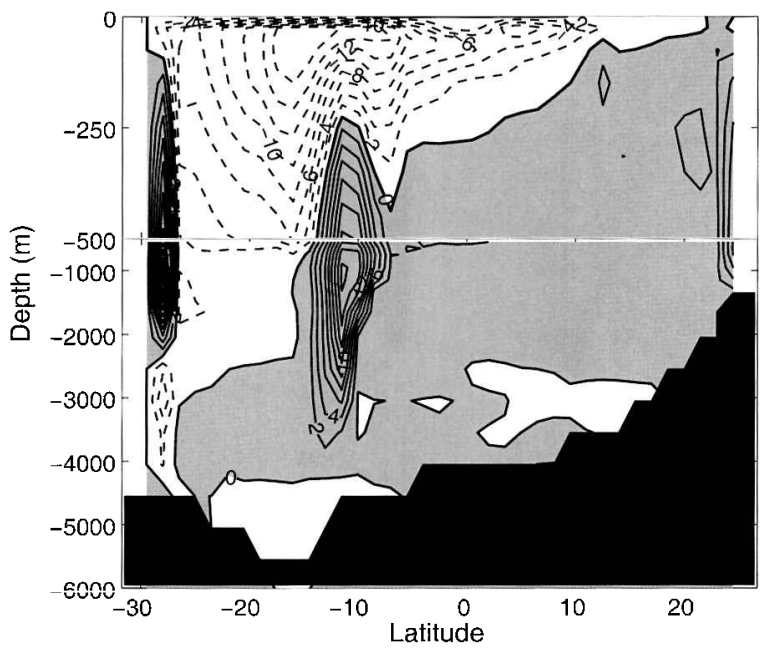

Figure 14. Meridional streamfunction of Sponge Expt. Contour interval 2 Sv; shaded areas and solid lines indicate positive values, dashed lines negative values, thick lines represent $0 \mathrm{~Sv}$. The circulation is such that high values are to the right (clockwise around a high).

\section{a. Boundary conditions}

An optimization is carried out with closed southern and eastern boundaries, where sponge layers are placed. The experiment is initialized with the final state of Spin-up II and is referred to as Sponge Expt. (Table 1). This experiment is the exact analog to the reference experiment of LM97 (their Run B), and the resultant meridional overturning (Fig. 14) is similar. However, our solution shows a very strong (16 Sv) clockwise overturning cell in the sponge near the Indonesian Archipelago; a similar one is seen in the southern sponge layer. The numerical formulation of the model, most likely the choice of a "C" grid, appears to amplify the tendency to form artificial overturning cells in sponge layers (see LM97). Our standard experiment shows that the solution with open boundaries behaves much better numerically, which is yet another argument for replacing the closed-wall and sponge boundary conditions with open boundary conditions.

\section{b. Stability}

To prove that the optimized result is indeed a stable near-steady state, a 100-year forward integration is appended to the optimization (Stability Expt., Table 1). The model is initialized with the optimized hydrographic fields and open boundary conditions, and is forced with the optimized surface fluxes; notice that no additional restoring terms are employed in the surface forcing. There is little change in the dominant shallow overturning cell after 100 years (figure not shown), demonstrating that indeed a near-steady state has been found by the optimization.

We have stated repeatedly that the ITF plays a minor role in the heat and freshwater budgets of our model. To test this, we repeat the Stability Expt. but close off the ITF 


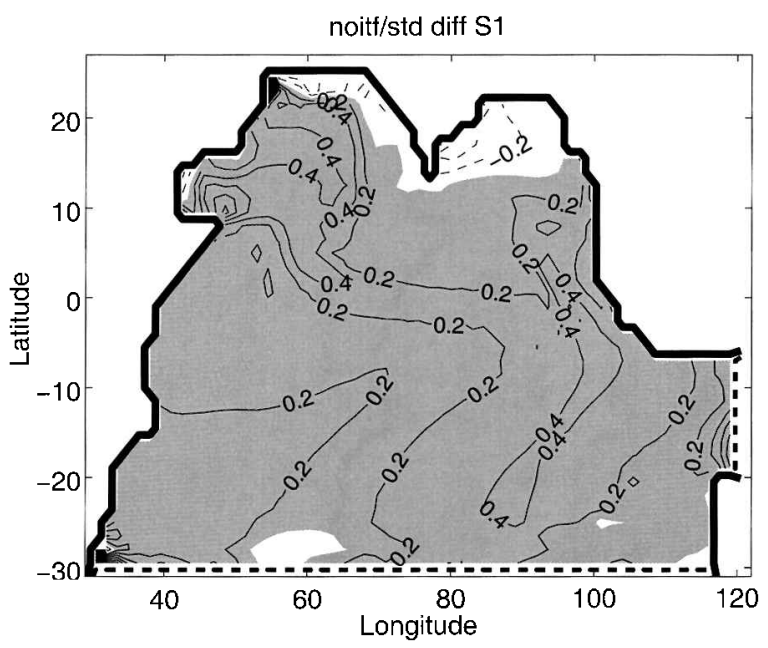

Figure 15. Surface salinity difference between the experiment with closed ITF and the P-E experiment, after 60 years. Shaded areas and solid lines indicate positive values, dashed lines negative values, contour interval 0.2 .

entirely (no mass, heat, and freshwater forcing, Expt. NOITF in Table 1; notice that we start from Expt. P-E, which we deem more realistic in its surface salinity budget). Figure 15 shows the difference in surface salinity between the NOITF and P-E experiments, after 60 years. While almost the entire domain gets saltier, pointing at the freshening by the ITF, the magnitude is relatively modest (generally 0.2 to 0.4 ). We expect that in an optimization experiment with the ITF closed, minor modifications in P-E and the southern boundary conditions would have compensated for the loss of ITF influence.

\section{Discussion and conclusions}

In every regional modeling study the question arises how the exchanges between the model domain and the rest of the World Ocean are formulated. Here, we have estimated the open boundary conditions from interior data, the model, and the model's adjoint. To our knowledge, it is the first ever application of this approach to a full-blown ocean general circulation model (GCM). A considerable investment into model development was required (more fully described in Marotzke et al., 1999; see also Stammer et al., 1997). Part of the motivation for this investment arose from the scientific focus of this paper: we have investigated aspects of the general circulation of the Indian Ocean, using climatological data and a model, and emphasizing the exchanges with the Southern and Pacific oceans. While we consider a specific application, this type of question is generic, and we thus describe a general strategy for model-data synthesis in a regional context.

The formal ill-posedness of the open-boundary problem in the primitive equations (Bennett, 1992) caused no practical difficulty here, presumably because of a mix of a robust (and diffusive enough) formulation and the estimation of the boundary condition 
parameters; the latter would eliminate solution features clearly in disagreement with interior data. Indeed, we find the solution with open boundaries numerically better behaved than a version with closed walls and buffer zones [the exact analog of the model of Lee and Marotzke, 1997 (LM97)]. Another indication that the open boundary formulation is successful comes from our estimate of the Agulhas Current and East Madagascar Current including its southward extension: Our model boundary cuts right across the center of the subtropical gyre in the southern Indian Ocean, yet there is a combined transport of $70 \mathrm{~Sv}$ leaving the model domain.

We find the overturning to be confined to the thermocline, wind-driven, and of strength $16 \mathrm{~Sv}$, consistent with the conclusions of LM97. Zonally integrated deep transport is weak, although somewhat stronger than that of LM97. Hence, it was not the artificially closed sidewalls and buffer zones in LM97 that caused the discrepancy between their results and those of Toole and Warren (1993) and Robbins and Toole (1997). As argued in the introduction, neither model resolution nor presence or absence of a seasonal cycle have changed the picture much. A critic has argued that the low observed oxygen values at mid-depth and high values at greater depth "prove" that a solution without deep integrated mass inflow is incorrect. We disagree with this conclusion. While the oxygen data, most notably at $18 \mathrm{~S}$ (Warren, 1981), clearly show that oxygen is imported from the south, this could well be accomplished by horizontal recirculations (deep western boundary currents entering, interior flow leaving at the same depth) or time-dependent flow, which all models agree are vigorous (Wacongne and Pacanowski, 1996; Garternicht and Schott, 1997; Lee and Marotzke, 1998). At issue is what can be inferred from zonal mean tracer distributions about zonal mean flows; we believe that a GCM inversion including tracers and timedependence is needed to rule out our solution as a valid fit to climatology.

Hence, the most likely candidates for explaining the discrepancies between the published Indian Ocean circulation schemes are the resolution of the climatology or the representativeness of a single hydrographic line. Work on the synthesis of the WOCE Indian Ocean dataset is ongoing, using essentially the model employed here, and is expected to answer this question.

We have defined generalizations of meridional heat and freshwater transports appropriate for flow with a net mass transport. They are dominated by the mass-balanced portion of the flow while the effect of the mass import from the ITF is small (see below). The meridional heat and freshwater transport divergences are well balanced by the surface heat flux, indicating near-steady state. The estimated meridional heat transport has a maximum of $0.8 \mathrm{PW}$ southward at $12 \mathrm{~S}$, close to the number of LM97 and well within the range of previous estimates (e.g., Toole and Warren, 1993; Macdonald and Wunsch, 1996; Robbins and Toole, 1997). Maximum freshwater transport is $0.29 \mathrm{~Sv}$ southward at 9S, which is essentially the value implied by the surface flux estimates by Oberhuber (1988). However, the latter seem largely compatible with the model and the salinity climatology; significant deviations from climatology occur only at the surface, where the model circulation appears to restore fronts that were smeared out by the climatology. 
The Indonesian throughflow (ITF) is widely believed to play an important role in the global thermohaline circulation (e.g., Gordon, 1986) and the heat and freshwater budgets of the Indian Ocean (e.g., Godfrey, 1996; Bray et al., 1997). Our results do not support these statements. The estimated ITF is $2.7 \mathrm{~Sv}$ westward, near the lower end of but well within the range of previous estimates (which go from 2.6 Sv eastward to $18.6 \mathrm{~Sv}$ westward, according to Table 1 in Godfrey's 1996 review). Moreover, the ITF influence on meridional heat and freshwater transports is negligible in our results. It is smaller than the difference between surface flux and ocean transport divergence, that is, smaller than the residual indicating deviation from steady state.

Notice that we do not wish to put in doubt the results of modeling studies showing significant impact of the ITF on modeled Indian Ocean or global circulations (e.g., Hirst and Godfrey, 1993). However, in global models the strength of the ITF depends considerably on subjective choices of topography in the Indonesian passages, as was described by Hirst and Godfrey (1993), who opted for a topography that permitted a very large ITF and hence had large influence. Here, we have addressed the reverse question: What strength ITF does a steady-state Indian Ocean hydrography require? The answer is clearly, not a large one. This is consistent with Macdonald and Wunsch's (1996) global inversion of hydrographic sections, in which assumed ITF strengths between 0 and $20 \mathrm{~Sv}$ did not produce any unacceptable regional consequences, and none at all globally. Notice that our results do not exclude larger ITF transports; while our model readily lends itself to tests analogous to those of Macdonald and Wunsch (1996), it would go beyond the scope of this paper to address this.

Acknowledgments. We thank Detlef Stammer, Ralf Giering, Chris Hill, and Tong Lee for their help in constructing the adjoint code, and Christophe Herbaut for providing us with his open boundary scheme. Bruno Ferron, Tong Lee, Carl Wunsch, and two anonymous reviewers gave helpful comments on an earlier version of the manuscript. Dimitris Menemenlis provided indispensablehelp with the revision of the paper. We are indebted to Mark Abbott (Oregon State University, OSU) who at a critical time in this project gave us access to the OSU Thinking Machine CM5, which is sponsored by NASA. Detlef Stammer provided the output from his optimized global model. This work was supported by the National Science Foundation through grants OCE-930135 and OCE9617570 .

\section{REFERENCES}

Baumgartner, A. and E. Reichel. 1975. The World Water Balance, Oldenbourg Verlag, Munich, $179 \mathrm{pp}$.

Bennett, A. F. 1992. Inverse Methods in Physical Oceanography, Cambridge University Press, 346 pp.

Bennett, A. F. and P. C. McIntosh. 1982. Open ocean modeling as an inverse problem: Tidal theory. J. Phys. Oceanogr., 12, 1004-1018.

Bogden, P. S., P. Malanotte-Rizzoli and R. Signell. 1996. Open-ocean boundary conditions from interior data: Local and remote forcing of Massachusetts Bay. J. Geophys. Res., 101, 6487-6500.

Bray, N. A., S. E. Wijffels, J. C. Chong, M. Fieux, S. Hautala, G. Meyers and W. M. L. Morawitz. 1997. Characteristics of the Indo-Pacific throughflow in the eastern Indian Ocean. Geophys. Res. Let., 24, 2569-2572. 
Döscher, R., C. W. Böning and P. Herrmann. 1994. Response of circulation and heat transport in the North Atlantic to changes in thermohaline forcing in northern latitudes: A model study. J. Phys. Oceanogr., 24, 2306-2320.

Fieux, M., C. Andrie, P. Delecluse, A. G. Ilahude, A. Kartavtseff, F. Mantisi, R. Molcard and J. C. Swallow. 1994. Measurements within the Pacific-Indian oceans throughflow region. Deep-Sea Res., 41, 1091-1130.

Fieux, M., R. Molcard and A. G. Ilahude. 1996. Geostrophic transport of the Pacific-Indian oceans throughflow. J. Geophys. Res., 101, 12,421-12,432.

Garternicht, U. and F. Schott. 1997. Heat fluxes of the Indian Ocean from a global eddy-resolving model. J. Geophys. Res., 102, 21,147-21,159.

Gates, W. L. and A. B. Nelson. 1975. A new (revised) tabulation of the Scripps topography on a 1 degree global grid, II, Ocean depths. Rep. R-1277-1-ARPA, Rand Corp., Santa Monica, CA.

Giering, R. and T. Kaminski. 1998. Recipes for adjoint code construction. ACM Trans. Math. Software, 24, 437-474.

Godfrey, J. S. 1996. The effect of the Indonesian throughflow on ocean circulation and heat exchange with the atmosphere: A review. J. Geophys. Res., 101, 12,217-12,237.

Gordon, A. L. 1986. Interocean exchange of thermocline water. J. Geophys. Res., 91, 5037-5046.

Gunson, J. and P. Malanotte-Rizzoli. 1996a. Assimilation studies of open-ocean flows. 1. Estimation of initial and boundary conditions. J. Geophys. Res., 101, 28,457-28,472.

—_ 1996b. Assimilation studies of open-ocean flows. 2. Error measures with strongly nonlinear dynamics. J. Geophys. Res., 101, 28,473-28,488.

Hellerman, S. and M. Rosenstein. 1983. Normal monthly wind stress over the world ocean with error estimates. J. Phys. Oceanogr., 13, 1093-1104.

Hirst, A. C. and J. S. Godfrey. 1993. The role of Indonesian throughflow in a global ocean GCM. J. Phys. Oceanogr., 23, 1057-1086.

Klinck, J. M. 1995. Thermohaline structure of an eddy-resolving North Atlantic model: The influence of boundary conditions. J. Phys. Oceanogr., 25, 1174-1195.

Lee, T. and J. Marotzke. 1997. Inferring meridional mass and heat transports of the Indian Ocean by fitting a general circulation model to climatological data. J. Geophys. Res., 102, 10,585-10,602.

_ 1998. Seasonal cycles of meridional overturning and heat transport of the Indian Ocean. J. Phys. Oceanogr., 28, 923-943.

Levitus, S. and T. P. Boyer. 1994. World Ocean Atlas 1994, Vol. 4, Temperature: NOAA Atlas NESDIS 4. U.S. Dept. of Commerce, Washington, D.C.

Levitus, S., R. Burgett and T. P. Boyer. 1994. World Ocean Atlas 1994, Vol. 3, Salinity: NOAA Atlas NESDIS 3. U.S. Dept. of Commerce, Washington, D.C.

Macdonald, A. M. and C. Wunsch. 1996. An estimate of global ocean circulation and heat fluxes. Nature, 382, 436-439.

Marotzke, J. 1992. The role of integration time in determining a steady state through data assimilation. J. Phys. Oceanogr., 22, 1556-1567.

Marotzke, J., R. Giering, K. Q. Zhang, D. Stammer, C. N. Hill and T. Lee. 1999. Construction of the adjoint MIT ocean general circulation model and application to Atlantic heat transport sensitivity. (in preparation).

Marotzke, J. and C. Wunsch. 1993. Finding the steady state of a general circulation model through data assimilation: Application to the North Atlantic Ocean. J. Geophys. Res., 98, 20,149-20,167.

Marshall, J., A. Adcroft, C. Hill, L. Perelman and C. Heisey. 1997a. A finite-volume, incompressible Navier-Stokes model for studies of the ocean on parallel computers. J. Geophys. Res., 102, 5753-5766. 
Marshall, J., C. Hill, L. Perelman and A. Adcroft. 1997b. Hydrostatic, quasi-hydrostatic, and non-hydrostatic ocean modeling. J. Geophys. Res., 102, 5733-5752.

Meyers, G., R. J. Bailey and A. P. Worby. 1995. Geostrophic transport of Indonesian throughflow. Deep-Sea Res., 42, 1163-1174.

Oberhuber, J. M. 1988. An Atlas Based on the COADS Data Set: The Budgets of Heat, Buoyancy and Turbulent Kinetic Energy at the Surface of the Global Ocean. Max-Planck-Inst. für Meteorol., Hamburg, Germany, 100 pp.

Orlanski, I. 1976. A simple boundary condition for unbounded hyperbolic flows. J. Comput. Phys., 21, 251-269.

Press, W. H., S. A. Teukolsky, W. T. Vetterling and B. P. Flannery. 1992. Numerical Recipes, 2nd ed., Cambridge University Press, 963 pp.

Redler, R. and R. Döscher. 1997. Effect of the overflows on the circulation in the subpolar North Atlantic: A regional model study. J. Geophys. Res., 102, 18,529-18,552.

Robbins, P. E. and J. M. Toole. 1997. The dissolved silica budget as a constraint on the meridional overturning circulation of the Indian Ocean. Deep-Sea Res., 44, 879-906.

Schiller, A. 1995. The mean circulation of the Atlantic Ocean north of 30 S determined with the adjoint method applied to an ocean general circulation model. J. Mar. Res., 53, 453-497.

Semtner, A. J., Jr. and R. M. Chervin. 1992. Ocean general circulation from a global eddy-resolving model. J. Geophys. Res., 97, 5493-5550.

Stammer, D., C. Wunsch, R. Giering, K. Q. Zhang, J. Marotzke, J. Marshall and C. Hill. 1997. The global ocean circulation estimated from TOPEX/POSEIDON altimetry and a general circulation model. Center for Global Change Science Report, 49, MIT, 40 pp.

Stevens, D. P. 1991. The open boundary condition in the United Kingdom fine-resolution Antarctic model. J. Phys. Oceanogr., 21, 1494-1499.

Toole, J. M. and B. A. Warren. 1993. A hydrographic section across the subtropical South Indian Ocean. Deep-Sea Res., 40, 1973-2019.

Tziperman, E., W. C. Thacker, R. B. Long, S.-M. Hwang and S. R. Rintoul. 1992. Oceanic data analysis using a general circulation model. Part II: A North Atlantic model. J. Phys. Oceanogr., 22, $1458-1485$.

Wacongne, S. and R. Pacanowski. 1996. Seasonal heat transport in a primitive equations model of the tropical Indian Ocean. J. Phys. Oceanogr., 26, 2666-2699.

Warren, B. A. 1981. Transindian hydrographic section at Lat. 18S: Property distributions and circulation in the South Indian Ocean. Deep-Sea Res., 28, 759-788.

- 1994. Driving the meridional overturning in the Indian Ocean. Deep-Sea Res., 41, 1349-1360.

Wijffels, S. E., R. W. Schmitt, H. L. Bryden and A. Stigebrandt. 1992. Transport of freshwater by the oceans. J. Phys. Oceanogr., 22, 155-162.

Wyrtki, K. 1988. Oceanographic Atlas of the International Indian Ocean Expedition, reprinted by Amerind Publishing Co., New Delhi, xi + 531 pp. 